\title{
Highly stable plasminogen activator inhibitor type one (VLHL PAI-1) protects fibrin clots from tissue plasminogen activator-mediated fibrinolysis
}

\author{
JERZY JANKUN ${ }^{1,2,3}$, ANSARI M. ALEEM ${ }^{1}$, STEVEN H. SELMAN ${ }^{2}$, \\ EWA SKRZYPCZAK-JANKUN ${ }^{1}$, WIESLAWA LYSIAK-SZYDLOWSKA ${ }^{3}$, \\ NICHOLAS GRAFOS ${ }^{4}$, HUGH J.L. FRYER ${ }^{4}$ and ROBERT S. GREENFIELD ${ }^{4}$
}

\begin{abstract}
${ }^{1}$ Urology Research Center, Department of Urology, ${ }^{2}$ Physiology, Pharmacology, Metabolism, and Cardiovascular Sciences, Health Science Campus, University of Toledo, Toledo, OH, USA; ${ }^{3}$ Department of Clinical Nutrition, Medical University of Gdansk, Gdansk, Poland; ${ }^{4}$ American Diagnostica Inc., Stamford, CT, USA
\end{abstract}

Received August 3, 2007; Accepted September 4, 2007

\begin{abstract}
Plasminogen activator inhibitor-1 (PAI-1) is the major specific inhibitor of tissue-type plasminogen activator (tPA) which mediates fibrin clot lysis through activation of plasminogen. Wild-type-PAI-1 (wPAI-1) is rapidly converted to the latent form (half-life of $\sim 2 \mathrm{~h}$ ) and loses its ability to inhibit tPA. We developed a very long half-life PAI-1 (VLHL PAI-1), a recombinant protein with a half-life $>700 \mathrm{~h}$ compared with wPAI-1. In this study, VLHL PAI-1 was assessed for its ability to inhibit clot lysis in vitro. Clot formation was initiated in normal plasma supplemented with tPA by the addition of either tissue factor or human recombinant FVIIa. Clot lysis time, monitored turbidimetrically in a microtiter plate reader, was determined at various concentrations of wPAI-1 and VLHL PAI-1. Both wPAI-1 and VLHL PAI-1 caused a significant increase in clot lysis time, although the latter was somewhat less effective at lower concentrations. The VLHL PAI-1, but not wPAI-1, maintained its anti-fibrinolytic activity after preincubation overnight at $37^{\circ} \mathrm{C}$. These studies demonstrate that VLHL PAI-1 is an effective inhibitor of fibrin clot degradation. Due to the high stability of VLHL PAI-1 compared with wPAI-1, this novel inhibitor of tPA-mediated fibrinolysis may have therapeutic applications for treating surgical and trauma
\end{abstract}

Correspondence to: Dr Jerzy Jankun, Urology Research Center, Health Science Campus, University of Toledo, Mail Stop 1091, 3065 Arlington, Toledo, OH 43614-5807, USA

E-mail: jerzy.jankun@utoledo.edu or

Dr Robert Greenfield, American Diagnostica Inc., 500 West Avenue, Stamford, CT 06902-6360, USA

E-mail: rgreenfield@amdiag.com

Key words: fibrin clot, tissue plasminogen activator, very long half-life, plasminogen activator inhibitor type one, FVIIa patients when used directly or in conjunction with the procoagulant recombinant FVIIa.

\section{Introduction}

Hemostasis depends on a delicate balance of the coagulation and fibrinolytic systems. An abnormality in either system can lead to excessive bleeding or clotting. The blood coagulation system minimizes blood loss at the site of injury by generating a plasmin-mediated insoluble fibrin clot. Following blood vessel repair, fibrinolytic enzymes dissolve the clot to restore blood flow. Fibrinolysis is finely regulated by the balance between tissue plasminogen activator (tPA), which activates plasminogen to plasmin, plasminogen activator inhibitor type 1 (PAI-1), which inhibits tPA (1), and $\alpha(2)$-antiplasmin $[\alpha(2) A P]$, which inhibits plasmin. Thus, the use of potent blood clotting agents without systemic activation of the coagulation cascade can be beneficial for patients suffering uncontrolled bleeding from conditions such as hemorrhagic disorders or severe trauma. Hemorrhage is a major cause of death of victims of accident- or battlefield-related traumas $(2,3)$. Recombinant factor VIIa (rFVIIa) is one of the more effective treatments for controlling hemorrhage in hemophilia. It has also been used to treat massive bleeding in trauma patients. According to Martinowitz et al (4) and Martinowitz and Michaelson (5), controlled animal trials, small case series, and anecdotal case reports suggest that the use of rFVIIa may slow and control massive bleeding during trauma, and hence prolong survival and reduce mortality. Furthermore, the survival rate of patients suffering from uncontrolled bleeding treated with rFVIIa was 61\% (22/36) and was favorable when compared with a published series of similar, or less severe, trauma patients (range 30-57\%) (5). This effect is enhanced if the clot is protected from tissue plasminogen activatorinduced fibrinolysis. In several studies it has been shown that PAI-1 protects fibrin clots from tPA-mediated plasmin degradation in a dose-dependent manner (6-11).

Active PAI-1 is quickly inactivated by a rapid, spontaneous conversion to the latent form (half-life 1-2 h) limiting its 
therapeutic potential. Active PAI-1 has a reactive center loop (RCL) that contains a P1-P1' site which extends from the body of the molecule. This site binds to and inactivates tPA. In latent PAI-1 this loop is retracted and becomes inserted between the A 3 and A5 strands of the corresponding B-sheet, making the P1-P1' site inaccessible to tPA $(12,13)$. By restricting the movement of the A3 and A5 strands, as well as limiting the flexibility of the helix D region, it is possible to prevent insertion of the reactive loop between A3 and A5 and hence extend the half-life of PAI-1 (14).

Based on the known structure of active PAI-1, we identified amino acids that can be substituted with cysteine residues to produce disulfide bridges that constrain PAI-1 into a stable form that remains active for a prolonged period of time. We previously identified seven possible cysteine mutants (two- to six-point mutations, with an even number of cysteines) at the top and bottom portions of strands A3 and A5 and within the helix D region, or by a combination thereof, as potential candidate sites. The longest lasting half-life was observed for a $\beta$-sheet-top PAI-1 mutant (two-point mutations). We measured its activity for up to $700 \mathrm{~h}$ and determined that the half-life for this mutant could reach $>6,900 \mathrm{~h}(14,15)$. In this study, we studied the ability of the VLHL PAI-1 mutant to inhibit tPA-mediated clot lysis in an in vitro model.

\section{Materials and methods}

Expression of VLHL PAI-1. The mutation of two amino acids (Gln197 $\rightarrow$ Cys, Gly355 $\rightarrow$ Cys) produced VLHL PAI-1 with a very long half-life $>700 \mathrm{~h}$. Construction of this protein was previously described (14). Briefly, the cDNA encoding PAI-1 was excised from the VLHL PAI-1 plasmid as a NdeI/Xho fragment. The PCR product of VLHL PAI-1 NdeI/XhoI fragment was ligated into the pFastbac plasmid, which contains a 6 His purification tag. To confirm mutations, the VLHL PAI-1 DNA construct was sequenced (MWG Biotech Inc., Mendenhall Oaks Parkway, NC).

Purification of VLHL PAI-1. VLHL PAI-1 was expressed and purified as described previously (16). Briefly, recombinant bacmid virus containing VLHL PAI-1 DNA was used to transfect Sf9 cells derived from Spodoptera Frugiperda (Fall Armyworm) using cellfectin reagent (Invitrogen) based on the manufacturer's instructions. The virus was then used to infect Sf9 cells $\left(\sim 2 \times 10^{6} / \mathrm{ml}\right)$ on a large scale (1 liter of cell culture in a 2.8 liter Fernbach flask). The flasks were incubated in a rotary incubator for $72 \mathrm{~h}$ at $27^{\circ} \mathrm{C}$. The cells were harvested, lysed by two freeze-thaw cycles, and the lysate was centrifuged at 3,000 x g for $20 \mathrm{~min}$ to pellet cellular debris. The supernatant was transferred to a fresh tube for purification.

The supernatant was loaded onto a nickel resin-packed column (Invitrogen, Carlsbad, CA) with a peristaltic pump (GradiFrac system, Pharmacia Biotech) at a flow rate of $0.8 \mathrm{ml} / \mathrm{min}$. The column was washed with buffer containing $40 \mathrm{mM}$ imidazole in native buffer $\left(50 \mathrm{mM} \mathrm{NaH} \mathrm{PO}_{4}, 0.5 \mathrm{M}\right.$ $\mathrm{NaCl}, \mathrm{pH} 8.0$, containing protease inhibitors) at a flow rate of $1 \mathrm{ml} / \mathrm{min}$ until A280 reached baseline. The protein was then eluted using a gradient of $40-250 \mathrm{mM}$ imidazole in native buffer at a flow rate of $1 \mathrm{ml} / \mathrm{min}$. The peak fractions were dialyzed to remove imidazole, concentrated, and used for further analysis. Unused purified protein was stored at $-80^{\circ} \mathrm{C}$

SDS-PAGE analysis. Electrophoresis of proteins was performed at room temperature using 4-12\% SDS-polyacrylamide gradient gels under non-reducing conditions. Gels were stained with Colloidal Coomassie Blue (Invitrogen, Grand Island, NY).

Chromogenic assay of VLHL PAI-1 activity. The activity of PAI-1 was assessed by adding equal volumes of VLHL PAI-1; $50 \mu \mathrm{l}$ diluted in $\mathrm{H}_{2} \mathrm{O}$ from a stock of $1.0 \mathrm{mg} / \mathrm{ml}$ in $20 \mathrm{mM}$ HEPES, $250 \mathrm{mM}$ imidazole, $\mathrm{pH} 8.0$ to a concentration of $0.06 \mathrm{mg} / \mathrm{ml}$ ) and HMW uPA (American Diagnostica Inc., Stamford, CT) diluted from $1.0 \mathrm{mg} / \mathrm{ml}$ to $0.3 \mathrm{mg} / \mathrm{ml}$ in water. Following a 15-min incubation at room temperature, $50 \mu 1$ of chromogenic uPA substrate (Spectrozyme ${ }^{\circledR}$ uPA, American Diagnostica Inc.), to a final concentration of $1.7 \mathrm{mM}$. Immediately following substrate addition, the absorbance at $405 \mathrm{~nm}$ was determined in a 96-well plate reader (VersaMax ${ }^{\mathrm{TM}}$ tunable microplate reader, Molecular Devices, Sunnyvale, CA) and was monitored every $20 \mathrm{sec}$ for $50 \mathrm{~min}$ with uPA alone or with wPAI-1/uPA as controls.

Determination of time of fibrinolysis. Each well of a 96-well plate was filled with $100 \mu 1$ of pooled normal human plasma, $20 \mu 1$ of tPA $(1.2 \mu \mathrm{g} / \mathrm{ml}), 40 \mu 1$ tissue factor $(\mathrm{TF}) / \mathrm{Ca}^{2+}$ (reagent ref no. 824 from American Diagnostica Inc.; diluted 1:100), $20 \mu 10.15 \mathrm{M} \mathrm{NaCl}$ or rFVIIa (from the same supplier and in the same buffer), and $20 \mu \mathrm{l}$ of wPAI-1 or VLHL PAI-1 mutant in different concentrations $(0.1,1.0,10.0 \mu \mathrm{g} / \mathrm{ml})$. For inactivation studies, wPAI- 1 and VLHL PAI- 1 were incubated at $37^{\circ} \mathrm{C}$ overnight to induce conversion of wPAI-1 to the latent form. Clot formation and dissolution was monitored at $405 \mathrm{~nm}$ every $45 \mathrm{sec}$ at $37^{\circ} \mathrm{C}$ using a SpectroMax Spectrophotometric plate reader (Molecular Devices). In this assay, clot formation is indicated by an increase in OD. Over time, the OD decreases due to clot dissolution by fibrinolytic processes, which reaches the initial OD when the clot has completely dissolved.

The areas under the fibrin aggregation curves were measured (Fig. 3). For the overall hemostatic potential determination $\left(\mathrm{OHP}_{\mathrm{TPA}}\right)$, plasma samples were mixed with rFVIIa or tissue factor and tPA. For the overall coagulation potential meaurements $\left(\mathrm{OCP}_{\text {control }}\right)$, plasma samples were mixed with rFVIIa or tissue factor as described above. For overall coagulation potential for PAI-1 $\left(\mathrm{OCP}_{\mathrm{PAI}-1}\right)$, tPA, rFVIIa or tissue factor and different concentration of wPAI-1 or VLHL PAI-1 were added. The overall clot protecting potential (OCPP) or inhibition of clot lysis was calculated as:

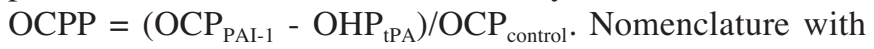
some modifications are used as in ref. 17.

\section{Results and discussion}

VLHL PAI-1 characterization. As shown in Fig. 1, the single-step purification of VLHL PAI-1 yielded a $\sim 95 \%$ pure protein $(\sim 10 \mathrm{mg}$ from 1 liter of cell culture medium) as determined by densitometry of the SDS-PAGE gel bands. 


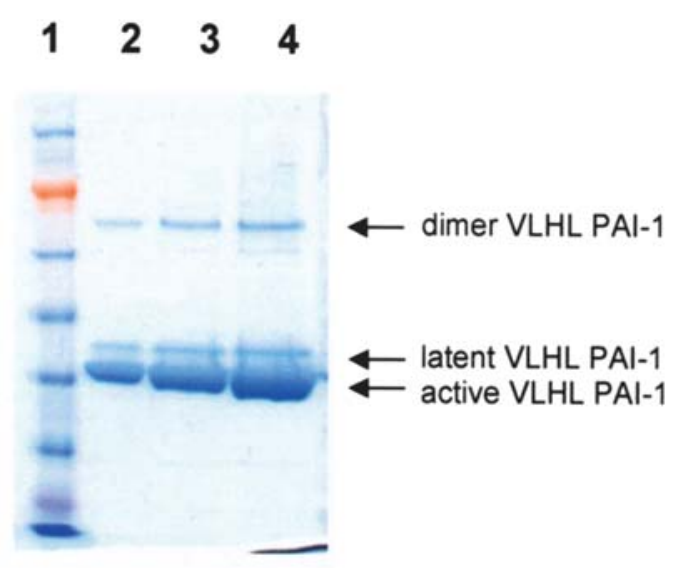

Figure 1. SDS-PAGE analysis of purified VLHL PAI-1. Different amounts of purified VLHL PAI-1 were separated on a 4-12\% SDS-polyacrylamide gel, which was subsequently stained with Coomassie blue. Lane 1: protein standards (from top, 191, 97, 64, 51, 39, 28, 19, and $14 \mathrm{kDa}$ ); lanes 2-4: 2, 5, and $10 \mu \mathrm{g}$, respectively, of purified VLHL PAI-1.

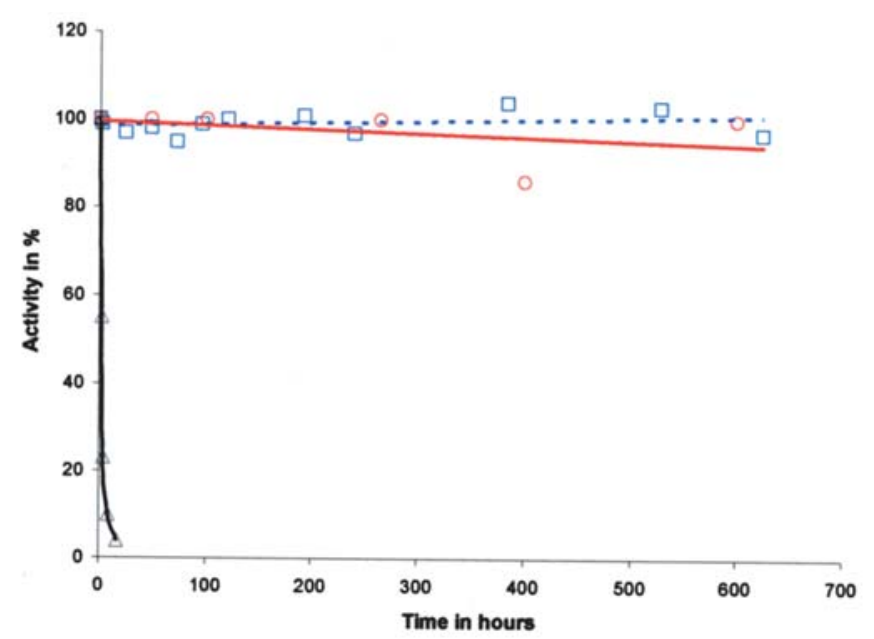

Figure 2. VLHL PAI-1 has a much longer half-life for activity compared with wPAI-1. Inhibitory activity (100\% represents activity at time 0 ) of wPAI-1 (triangle); intein-containing VLHL PAI-1 (squares, as reported previously) (15), and 6His-containing VLHL PAI-1 (open circles).
The heavily stained $\sim 40-\mathrm{kDa}$ band represents active VLHL PAI-1, the faint $\sim 45-\mathrm{kDa}$ band is the latent form of VLHL PAI-1, and the weakly stained $\sim 75-\mathrm{kDa}$ band is a dimerized VLHL PAI-1. Identity of these protein bands was confirmed by liquid chromatography/mass spectrometry as previously reported $(16,18)$.

Our initial VLHL PAI-1 construct contained the intein purification tag, which was expressed in E. coli. The yield of protein from this preparation was relatively low with only 1-2 mg from 1 liter of cell culture medium (15). A new vector containing a $6 \mathrm{His}$ tag was constructed and expressed in insect cells to increase the protein yield. As shown in Fig. 2 the half-life of the active form of the 6His tag VLHL PAI-1 construct was approximately the same as for the earlier construct $(\sim 700 \mathrm{~h})(15)$. In all experiments, the activity of VLHL PAI-1 was normalized to VLHL PAI-1 protein concentrations.

Inhibition of fibrinolysis by VLHL PAI-1. Clot lysis by endogenous enzymes from pooled plasma takes $\sim 10-12 \mathrm{~h}$ (19). To increase the speed of normal clot lysis and thus facilitate the analysis of the clot-protecting ability of VLHL PAI-1, tPA was added to pooled normal plasma prior to initiation of clot formation.

As indicated by the data displayed in Fig. 4, addition of wPAI-1 or VLHL PAI-1 increased the time of clot lysis. VLHL PAI- 1 at $0.1 \mu \mathrm{g} / \mathrm{ml}$ was slightly less potent than wPAI-1. The lower activity of VLHL PAI- 1 can be explained by Hagglof et al, who hypothesized that PAI-1s with Cys mutations in the A3 and A5 strands have reactive center loops that form a bulge on one side of the protein, which is shifted to the opposite side resulting in a more spherical molecule than active wPAI-1, where RCL is extended from the top of the protein molecule (20). This alteration in structure may lower the affinity of VLHL PAI-1 for tPA.

VLHL PAI-1 retained its clot-protecting abilities even with prolonged incubation. As shown in Fig. 4, overnight incubation $(14 \mathrm{~h})$ of wPAI-1 resulted in slight $(10.0 \mu \mathrm{g} / \mathrm{ml})$, significant $(1.0 \mu \mathrm{g} / \mathrm{ml})$ and complete $(0.1 \mu \mathrm{g} / \mathrm{ml})$ loss of clot protection abilities, while the clot-protective properties of VLHL PAI-1 remained relatively unchanged. Time to

\section{A}

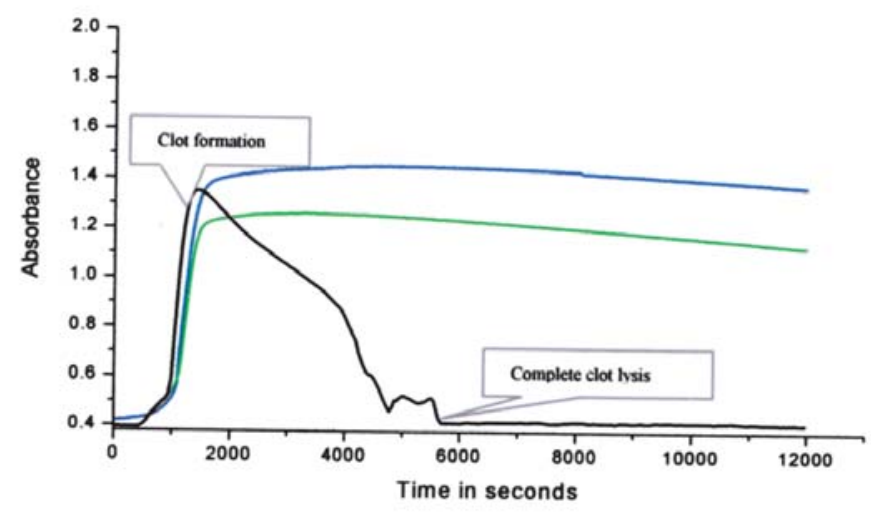

B

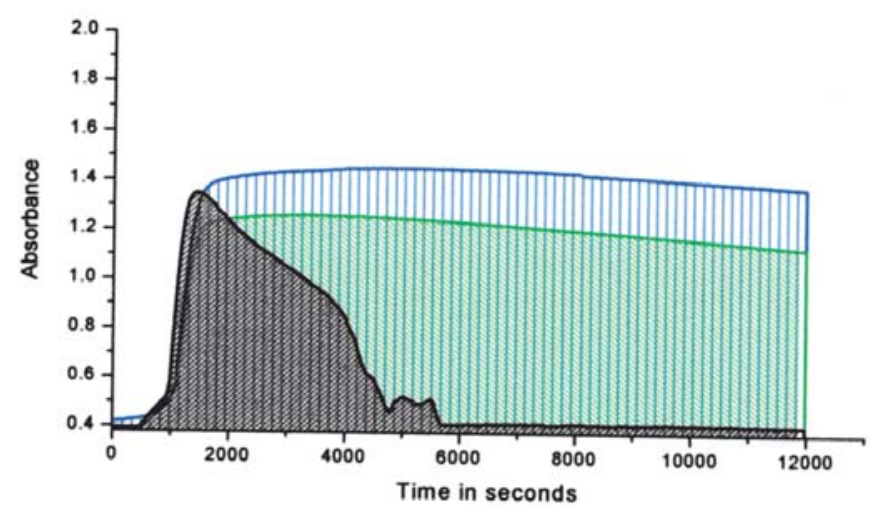

Figure 3. Spectrophotometric tracing of clot formation and lysis. Clot inducing factors were added to normal human plasma at time zero. (A) Plasma plus tPA (black line); plasma alone (green line); and tPA and PAI-1 added to plasma (blue line). (B) The areas under the fibrin aggregation curves were calculated as the sum of absorbance multiplied by the time step. 


\section{No incubation}
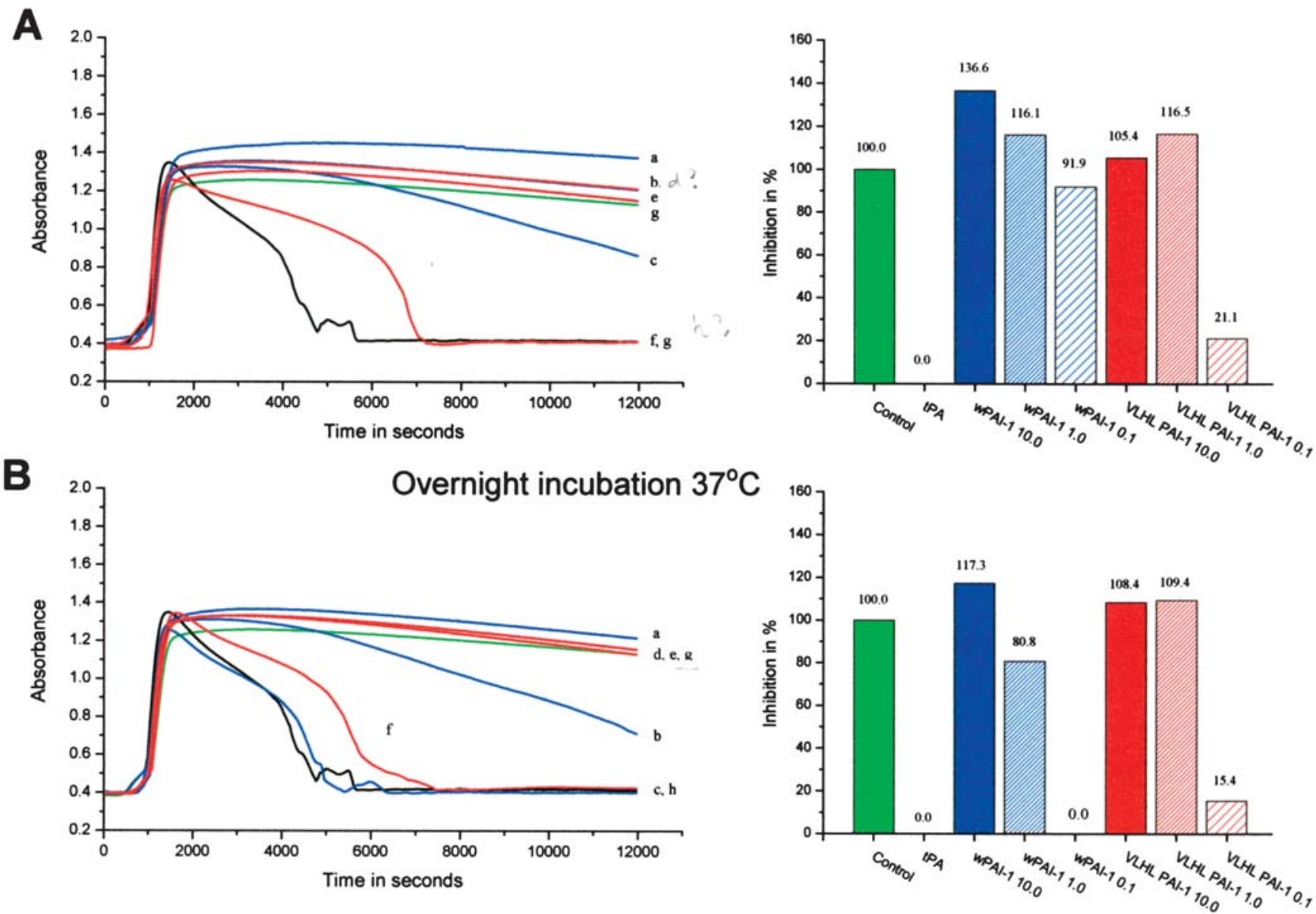

Figure 4. Overnight incubation of VLHL PAI-1 does not decrease its ability to inhibit fibrinolysis. (A) wPAI-1 and VLHL PAI-1 without preincubation. (B) wPAI-1 and VLHL PAI-1 incubated at $37^{\circ} \mathrm{C}$ overnight prior to addition to plasma. Spectrophotometric tracing of clot formation and lysis (left column) for no tPA or PAI-1 added (green, g), tPA added without PAI-1 (black, h), or tPA added with various concentrations of wPAI-1 (blue: a, $10 \mathrm{ng} / \mathrm{ml} ; \mathrm{b}, 1.0 \mathrm{ng} / \mathrm{ml}$; c, $0.1 \mathrm{ng} / \mathrm{ml}$ ), or VLHL PAI-1 (red: d, $10 \mathrm{ng} / \mathrm{ml} ; \mathrm{e}, 1.0 \mathrm{ng} / \mathrm{ml} ; \mathrm{f}, 0.1 \mathrm{ng} / \mathrm{ml}$ ). The overall clot protecting potential (OCPP) is expressed as percent (right column; color scheme as above).

complete lysis was $~ 5,500 \mathrm{sec}$ for tPA alone and for tPA + $0.1 \mu \mathrm{g} / \mathrm{ml}$ wPAI-1, while it was unchanged for TPA $+0.1 \mu \mathrm{g} /$ ml VLHL PAI-1 ( 7,200 sec). Lysis velocity of VLHL PAI-1 $(1.0 \mu \mathrm{g} / \mathrm{ml}, 10.0 \mu \mathrm{g} / \mathrm{ml})$ remained unchanged. This indicates that VLHL PAI-1 was able to maintain an active form for a much longer period of time than wPAI-1.

In addition to its ability to block clot lysis induced by $\mathrm{TF} / \mathrm{Ca}^{2+}$, VLHL PAI-1 also blocked clot lysis initiated by rFVIIa (data not shown).

While the ability of PAI-1 to inhibit tPA-mediated fibrinolysis has long been known, the therapeutic potential of wPAI-1 has not been thoroughly investigated. This may be due in part to its rapid loss of protease inhibitory activity as a result of conversion to the latent form. Our findings suggest that VLHL PAI-1 may be a highly effective anti-fibrinolytic agent with therapeutic potential. The disulfide bridge of the recombinant VLHL PAI-1 prevents the reactive loop from embedding into the protein and thereby maintains the active conformation for a much longer period of time. VLHL PAI-1 was shown to be highly resistant to inactivation by heating at $37^{\circ} \mathrm{C}$. The improved pharmacokinetics suggest that VLHL PAI-1 may be an ideal candidate for therapeutic use in various bleeding conditions including hemophilia, trauma, tooth extraction, surgical injury as well as in rebleeding following initial hemostasis (21). The ability of VLHL PAI-1 to inhibit fibrinolysis, and thus stabilize a clot may prove helpful when administered systemically to assist in stemming blood flow during episodes of severe bleeding. Administration of recombinant FVIIa has proved highly effective in treating uncontrolled bleeding in hemophilia. rFVIIa is also being investigated in clinical studies as a rapid procoagulant for controlling severe bleeding in trauma and surgery. In this study, we showed that VLHL PAI-1 inhibits fibrinolytic degradation of fibrin clots formed by rFVIIa in vitro. It is possible that administering the combination of the antifibrinolytic VLHL PAI-1 along with the procoagulant rFVIIa would be a more effective therapeutic treatment than rFVIIa alone for controlling severe bleeding conditions.

VLHL PAI-1 may also be useful for treating patients with defective PAI-1 proteins. Spontaneous bleeding is rarely observed in patients with PAI-1 mutations or deficiencies, whereas moderate hemorrhaging of the knees, elbows, nose and gums are usually triggered by mild trauma. In these patients, however, menstrual bleeding may be severe, and 
prolonged bleeding after surgery is common (22-24). VLHL PAI-1 may be used for treating these patients under these conditions. Another potential application is the protective role of VLHL PAI-1 in reducing early mortality rate in lethal pneumococcal pneumonia. It has been found by Lim et al that administration of exogenous PAI-1 prevented lung injury and reduced mortality rate during severe pneumococcal preumonia in vivo (25).

In conclusion, these studies demonstrate that VLHL PAI-1 effectively protects fibrin clots from degradation by the fibrinolytic system. VLHL PAI-1 with its very long half-life offers possible therapeutic applications for surgery and trauma patients and for other patients where premature clot lysis may be dangerous to the individual.

\section{Acknowledgements}

We thank Dr R. Hart, President, American Diagnostica Inc. for discussions, for his support and the chemicals used in this study. We also thank Dr H. Hassouna (Michigan State University, East Lansing, MI, USA) for her valuable advice and in-depth discussions on this topic. This study was supported in-part by grants from American Diagnostica Inc., NIH CA90524 and CA109625, and Frank D. Stranahan Endowment Fund for Oncological Research.

\section{References}

1. Urano T, Ihara H, Suzuki Y, Takada $Y$ and Takada A: Coagulation-associated enhancement of fibrinolytic activity via a neutralization of PAI-1 activity. Semin Thromb Hemost 26: 39-42, 2000.

2. Holcomb JB, McMullin NR, Pearse L, et al: Causes of death in U.S. Special Operations Forces in the global war on terrorism: 2001-2004. Ann Surg 245: 986-991, 2007.

3. Sauaia A, Moore FA, Moore EE, et al: Epidemiology of trauma deaths: a reassessment. J Trauma 38: 185-193, 1995.

4. Martinowitz U, Kenet G, Segal E, et al: Recombinant activated factor VII for adjunctive hemorrhage control in trauma. J Trauma 51: 431-439, 2001.

5. Martinowitz U and Michaelson M: Guidelines for the use of recombinant activated factor VII ( $\mathrm{rFVIIa)}$ in uncontrolled bleeding: a report by the Israeli Multidisciplinary rFVIIa Task Force. J Thromb Haemost 3: 640-648, 2005.

6. Carr ME Jr, Krishnamurti C and Alving BM: Effect of plasminogen activator inhibitor-1 on tissue-type plasminogen activator-induced fibrinolysis. Thromb Haemost 67: 106-110, 1992.

7. Knabb RM, Chiu AT and Reilly TM: Effects of recombinant plasminogen activator inhibitor type 1 on fibrinolysis in vitro and in vivo. Thromb Res 59: 309-317, 1990.

8. Reilly CF, Fujita T, Hutzelmann JE, Mayer EJ and Shebuski RJ: Plasminogen activator inhibitor-1 suppresses endogenous fibrinolysis in a canine model of pulmonary embolism. Circulation 84: 287-292, 1991.
9. Reilly CF and Hutzelmann JE: Plasminogen activator inhibitor-1 binds to fibrin and inhibits tissue-type plasminogen activatormediated fibrin dissolution. J Biol Chem 267: 17128-17135, 1992.

10. Robbie LA, Booth NA, Croll AM and Bennett B: The roles of alpha 2-antiplasmin and plasminogen activator inhibitor 1 (PAI-1) in the inhibition of clot lysis. Thromb Haemost 70: 301-306, 1993.

11. Torr-Brown SR and Sobel BE: Attenuation of thrombolysis by release of plasminogen activator inhibitor type-1 from platelets. Thromb Res 72: 413-421, 1993.

12. Lawrence DA, Strandberg L, Ericson J and Ny T: Structurefunction studies of the SERPIN plasminogen activator inhibitor type 1 . Analysis of chimeric strained loop mutants. J Biol Chem 265: 20293-20301, 1990.

13. Verhamme I, Kvassman JO, Day D, et al: Accelerated conversion of human plasminogen activator inhibitor-1 to its latent form by antibody binding. J Biol Chem 274: 17511-17517, 1999.

14. Chorostowska-Wynimko J, Skrzypczak-Jankun E and Jankun J: Plasminogen activator inhibitor type-1 controls the process of the in vitro sprout formation. J Physiol Pharmacol 55 (suppl 3): 49-56, 2004.

15. Chorostowska-Wynimko J, Swiercz R, Skrzypczak-Jankun E, Wojtowicz A, Selman SH and Jankun J: A novel form of the plasminogen activator inhibitor created by cysteine mutations extends its half-life: relevance to cancer and angiogenesis. Mol Cancer Ther 2: 19-28, 2003.

16. Jankun J, Aleem AM, Specht Z, et al: PAI-1 induces cell detachment, downregulates nucleophosmin (B23) and fortilin (TCTP) in LnCAP prostate cancer cells. Int J Mol Med 20: 11-20, 2007.

17. Antovic JP, Antovic A, Sten-Linder M, Wramsby M and Blomback M: Overall hemostatic potential (OHP) assay-a possible tool for determination of prothrombotic pattern in FXII deficiency. J Thromb Haemost 2: 2058-2060, 2004.

18. Jankun J, Specht Z, Szkudlarek M, et al: Plasminogen activator inhibitor-1 is locked in active conformation and polymerizes upon binding ligands neutralizing its activity. Int J Mol Med 17: 437-447, 2006.

19. Stief TW, Frohlich S and Renz H: Determination of the global fibrinolytic state. Blood Coagul Fibrinolysis 18: 479-487, 2007.

20. Hagglof P, Bergstrom F, Wilczynska M, Johansson LB and Ny T: The reactive-center loop of active PAI-1 is folded close to the protein core and can be partially inserted. J Mol Biol 335: 823-832, 2004.

21. Minowa H, Takahashi Y, Tanaka T, et al: Four cases of bleeding diathesis in children due to congenital plasminogen activator inhibitor-1 deficiency. Haemostasis 29: 286-291, 1999.

22. Kuhli C, Luchtenberg M, Scharrer I and Hattenbach LO: Massive subhyaloidal hemorrhage associated with severe PAI-1 deficiency. Graefes Arch Clin Exp Ophthalmol 243: 963-966, 2005 .

23. Takahashi Y, Tanaka T, Minowa H, et al: Hereditary partial deficiency of plasminogen activator inhibitor-1 associated with a lifelong bleeding tendency. Int J Hematol 64: 61-68, 1996.

24. Zhang ZY, Wang ZY, Dong NZ, Bai X, Zhang W and Ruan CG: A case of deficiency of plasma plasminogen activator inhibitor-1 related to Ala15Thr mutation in its signal peptide. Blood Coagul Fibrinolysis 16: 79-84, 2005.

25. Lim JH, Stirling B, Derry J, et al: Tumor suppressor CYLD regulates acute lung injury in lethal streptococcus pneumoniae infections. Immunity 27: 349-360, 2007. 